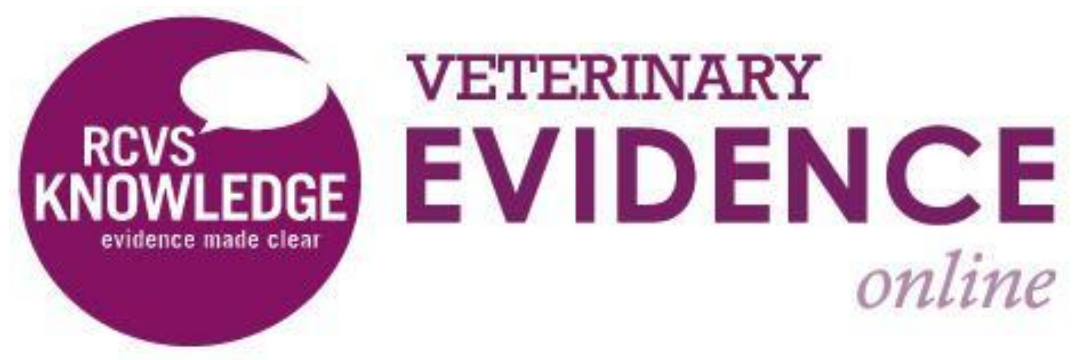

\title{
An interview with Dr Steve Budsberg
}

Richard Evans

Editor-in-chief, Veterinary Evidence

ISSN: 2396-9776

Published: 27 Jan 2016

in: Vol 1, Issue 1

DOI: http:// 10.18849/ve.v1i1.21 
Veterinary Evidence Editor-in-Chief Rich Evans caught up with long-time evidence-based veterinary medicine advocate Dr. Steve Budsberg at this year's American College of Veterinary Surgeons Symposium and took the opportunity to ask Dr. Budsberg his take on some of the current issues in EBVM. Dr. Budsberg, an orthopedic surgeon at the University of Georgia, USA, is a past president and charter member of the Evidence-base Veterinary Medical Association and on the program committee for Veterinary Evidence Today: The 2016 EBVM Network Conference, to be held in November, in Edinburgh.

VE: Steve, you've been working in EBVM a long time, trying to expand its use among academics and practitioners. What are the current roadblocks to implementing EBVM in practice?

Budsberg: I think it is multipronged problem. First there is a misunderstanding of the terminology. Second, quite frankly, we don't have a lot of solid clinical data to support most therapies or treatments we currently use in practice (that's frustrating, but it is changing), and the final road block is the aspect of change. Veterinarians must be encouraged (and shown the benefits) of including the use of EBVM in their day to day practice lives. This will be a slow progression and will inevitably take time.

VE: Can you give some examples of misunderstood terms?

Budsberg: The first misunderstanding is that people think if there is no published data then they can't use a treatment. Second they believe the EBVM will take away their options of which therapies to choose. Finally they believe that EBVM is only usable in academia and is impractical for them to integrate into their daily practice.

VE: You became a vet before the term "evidence-base" was coined. When in your career did you first get interested in EBVM?

Budsberg: Well, I would not say I was thinking about EBVM at the time, but when I was a third year veterinary student (1981) at Washington State I was introduced to a newly designed force plate system. By modern standards it was crude, but it caught my eye as a method of quantification of force placed on the ground by a dog.

Little did I know that those first ideas would end up defining much of my professional activities and my path into gait analysis. Dr. David Eddy first published the term "evidence-based" in March, 1990, in an article in the Journal of the American Medical Association that laid out the principles of evidence-based guidelines, and in 1996, Dr. David Sackett and others clarified the definition of evidence-based medicine as "the conscientious, 
explicit and judicious use of current best evidence in making decisions about the care of individual patients."

VE: Did you have any faculty support for your EBVM leanings?

Budsberg: As a surgical resident, I met Dr. Robert Soutas-Little at Michigan State University in 1985 and with his help and the support of Drs. Curtis Probst and Charlie DeCamp I started my journey into the quantification of outcomes in veterinary orthopedics and thus started my introduction into what is now called EBVM.

Then, I had a client (Dr. Mark Ebell) who was a physician/scientist in the late 1990s who was a national advocate of EBM and with his help, I started to meld my work in the quantification of clinical outcome measures with the concepts and methodologies of EBVM.

VE: What are the current problems with EBVM teaching and methodology?

Budsberg: Ignorance and inertia.

VE: Do you mean that instructors don't understand EBVM?

Budsberg: Well most instructors in Veterinary Curriculum have the same misunderstanding that I listed earlier. They are not versed in the terminology and the basic tenants of what EBVM is and how it can be applied to their particular field.

VE: If you could teach veterinary students one thing about EBVM, what would it be?

Budsberg: I would like students to consider how clinical treatments or clinical recommendations came into practice prior to it being taught.

It is understandable to say that we have years of clinical practice supporting therapies but it is also nice to know when there is really good data from well-designed studies that confirm a currently recommended therapy.

VE: You've written a lot about the power (i.e., sample size) of veterinary studies. Realistically, what can be done to increase the power of studies?

Budsberg: Again, it's all about education: understanding of the importance of knowing more about a study 
than whether or not $p \leq 0.05$. One must understand Type I and Type II error. Practitioners need to get their heads around the use of NNT for effect size, and researchers must learn other methods of study design such as non-inferiority studies and so on.

VE: What needs to be done to make EBVM part of the mainstream veterinary discussion?

Budsberg: Education and leading by example. Certainly this is a change in culture and that takes time but people have to carry the banner onward and it will change with time.

VE: Does this mean educating practicing vets or students?

Budsberg: Yes it means educating everyone along the continuum from students (not limited to professional students), private practitioners as well as clinical educators.

VE: What proportion of your clinical decisions are evidence based?

Budsberg: EBVM probably influences 30 to 40 percent of my clinical decisions

VE: That seems like a high percentage given the relative lack of evidence. Is that because some of your orthopedic procedures are supported by strong evidence?

Budsberg: Exactly, given the recent published manuscripts on TPLO treatment of cruciate injuries as well as the large amount of published studies on the use of NSAIDs, and high N-3 fatty acid diets in the treatment of canine osteoarthritis I feel I have some excellent data to support many of my clinical decisions.

VE: You are an orthopedist: what areas of orthopedics are lacking in evidence?

Budsberg: In orthopedics, I think we need more clinical data on the potential methods of treating joint disorders such as hip laxity, and fragmented coronoid processes. 


\section{Intellectual Property Rights}

Authors of Commentaries submitted to RCVS Knowledge for publication will retain copyright in their work, but will be required to grant to RCVS Knowledge an exclusive license of the rights of copyright in the materials including but not limited to the right to publish, re-publish, transmit, sell, distribute and otherwise use the materials in all languages and all media throughout the world, and to license or permit others to do

so.

Authors will be required to complete a license for publication form, and will in return retain certain rights as detailed on the form.

Veterinary Evidence and EBVM Network are RCVS Knowledge initiatives. For more information please contact us at editor@veterinaryevidence.org.

RCVS Knowledge is the independent charity associated with the Royal College of Veterinary Surgeons (RCVS). Our ambition is to become a global intermediary for evidence based veterinary knowledge by providing access to information that is of immediate value to practicing veterinary professionals and directly contributes to evidence based clinical decision-making.

\section{www.veterinaryevidence.org}

RCVS Knowledge is a registered Charity No. 230886. Registered as a Company limited by guarantee in England and Wales No. 598443.

Registered Office:

Belgravia House

62-64 Horseferry Road London SW1P 2AF 